\section{Optical Frequency Domain Imaging and Intravascular Ultrasound for Adult-Onset Kawasaki Disease in the Sub-Acute Phase}

Toshiki Kuno, MD, PhD; Akimichi Shibata, MD; Masaki Kodaira, MD, PhD;

Yohei Numasawa, MD, PhD

$\mathbf{W}$ e previously reported on an 18-year-old patient with adult-onset Kawasaki disease (KD). ${ }^{1}$ Given that the coronary computed tomography angiography showed a coronary artery aneurysm (CAA) along the left anterior descending artery (LAD) and the patient complained of chest pain, we performed coronary angiography 5 months after onset. Angiography showed the CAA along the LAD and the irregular vessel wall of the right coronary artery (RCA; Figure A,B). Notably, optical frequency domain imaging (OFDI; FastView, Terumo) and intravascular ultrasound (IVUS; ViewIt, Terumo) showed intimal thickening without calcification in the CAA along the LAD (Figure C,D, Movie S1). In addition, OFDI and IVUS indicated diffuse intimal thickening along with the aneurysmal changes in the RCA (Figure E,F). The fractional reserve flow in the LAD was 0.89 . OFDI and IVUS of KD usually show severe calcification in the later phase. ${ }^{2}$ In the present case, however, CAA with intimal thickening without calcification was seen because coronary angiography was performed in the subacute phase. This is extremely rare because most patients with KD are children, and we cannot perform intravascular imaging for children in that phase.

\section{Acknowledgment}

Japanese Red Cross Ashikaga Hospital, cardiology wards.

Received June 19, 2018; revised manuscript received August 1, 2018; accepted August 21, 2018; J-STAGE Advance Publication released online September 29, 2018 Time for primary review: 33 days

Department of Medicine, Mount Sinai Beth Israel Medical Center, New York, NY (T.K.), USA; Department of Cardiology (T.K., M.K., Y.N.), Department of Pediatrics (A.S.), Japanese Red Cross Ashikaga Hospital, Ashikaga, Japan

Mailing address: Toshiki Kuno, MD, PhD, Department of Medicine, Mount Sinai Beth Israel Medical Center, First Avenue, 16th Street, New York, NY 10003, USA. E-mail: toshiki.kuno@mountsinai.org ISSN-1346-9843 All rights are reserved to the Japanese Circulation Society. For permissions, please e-mail: cj@j-circ.or.jp

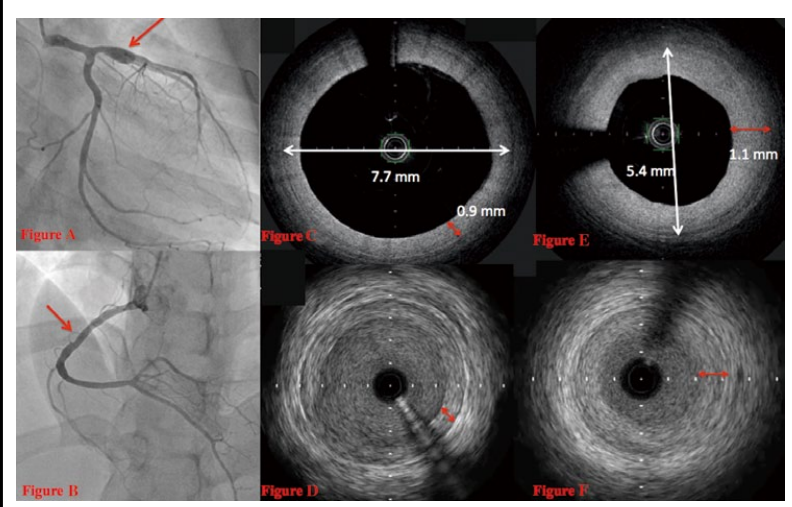

Figure. (A,B) Coronary angiography showing $(\mathbf{A})$ a coronary artery aneurysm (CAA) in the proximal left anterior descending artery (LAD; arrow) and ectatic changes in coronary arteries, on right anterior oblique-caudal view; and $(\mathbf{B})$ the irregular vessel wall of the right coronary artery (RCA; arrow), on left anterior oblique-cranial view. (C) Optical frequency domain imaging (OFDI) and (D) intravascular ultrasound (IVUS) showing a CAA with intimal thickening in the proximal LAD; $(\mathbf{C})$ white arrow: media-media, $7.7 \mathrm{~mm}$; red arrow: intima-media thickness, $0.9 \mathrm{~mm}$; 1 division of scale, $0.5 \mathrm{~mm}$; (D) red arrow, intima-media thickness; 1 division of scale, $1.0 \mathrm{~mm}$. (E) OFDI and (F) IVUS showing diffuse intimal thickening of the RCA; (E) white arrow: media-media, $5.4 \mathrm{~mm}$; red arrow: intima-media thickness, $1.1 \mathrm{~mm}$; 1 division of scale, $0.5 \mathrm{~mm}$; $(\mathbf{F})$ red arrow, intima-media thickness; 1 division of scale, $1.0 \mathrm{~mm}$.

\section{Disclosures}

The authors declare no conflicts of interest.

\section{References}

1. Kuno T, Shibata A, Kodaira M, Numasawa Y. Utility of coronary computed tomography angiography in the diagnosis and management of acute-phase adult-onset Kawasaki disease. Circ J 2018; 82: 3106-3107.

2. Kuramitsu S, Hiromasa T, Jinnouchi H, Domei T, Shirai S, Ando K. Usefulness of rotational atherectomy with optical frequency domain imaging guidance for severe calcified coronary lesions after Kawasaki disease. Cardiovasc Interv Ther 2017; 32: $154-158$.

\section{Supplementary Files}

Supplementary File 1

Movie S1. Optical frequency domain imaging showing a coronary artery aneurysm with intimal thickening in the proximal left anterior descending artery.

Please find supplementary file(s);

http://dx.doi.org/10.1253/circj.CJ-18-0713 